\title{
Introduction of recognition for musculoskeletal disorders
}

\begin{abstract}
It has been reported that most of logistic workers suffered from MSDs in the lumbar region. Our previous studies attempted to determine the main characteristic of the MMH of skilled experts in order to recognize difference in posture between healthy and injured workers. Our recent study has tried to propose the method to prepare the warm-up exercise and evaluate the effect of exercise through results of clinical test. In this paper, the introduction of recognition for MSD and the limitation of research were reported to reduce the high ratio of occupational MSDs. We believe that our approach can be one of examples to solve clinical healthcare challenges.
\end{abstract}

Keywords: early detection, musculoskeletal disorder;, vertical ground reaction force
Volume 3 Issue | - 2017

\author{
Hieyong Jeong, Yuko Ohno \\ Department of Robotics \& Design for Innovative Healthcare, \\ Osaka University, Japan
}

Correspondence: Hieyong Jeong, Department of Robotics \& Design for Innovative Healthcare, Osaka University, Japan, Tel +8I-6-6879-26I4, Email h.jeong@sahs.med.osaka-u.ac.jp

Received: August II, 2017 | Published:September 25, 2017
Abbreviations: CoP, center of pressure; MMH, manual material handling; MSD, musculo skeletal disorder; vGRF, vertical ground reaction force.

\section{Introduction}

Increasingly, increasingly, engineering should be required to solve clinical healthcare challenges. Our authors try to bridge the gap between engineers and clinical workers and translate engineering knowledge into clinical applications in order to accelerate biomedical innovation, trial, and commercialization. In this paper, the introduction of recognition for MSD is reported and we believe that our approach can be one of the examples to solve several clinical healthcare challenges. Figure 1 shows an importance to recognize the difference in posture between skilled experts and unskilled novices during lifting. If such a difference exists, because it is possible to recognize the degree and phase of the difference in posture with the quantitative value, we expect to connect to reduce the current high ratio of occupational low back pain. However, although there is no doubt that body position affects the magnitude of the loads on the spine, there is existed the serious problem which no logistic worker knows whether the own current $\mathrm{MMH}$ is proper or not. Although there have been a lot of results related to measure the relationship between the $\mathrm{MMH}$ and the burden of the body, it is not easy for logistic workers to measure the MMH by themselves. That is the reason why most of the conventional sensing system is ill-suited to the actual logistic workplace. Computer vision system, such as motion capture system, requires the installation of multiple cameras inside the container and the method to track all of the markers. Wearable system, such as accelerometer and motion suit, requires a mounting system that does not interfere with the worker in terms of handling or discomfort. Furthermore, the most significant problem is that conventional sensing system has a difficulty in measuring the influence of various conditions between the load and the human body on the lifting posture.

Focusing on these several points, we proposed the use of CoP trajectories in one of our previous studies. ${ }^{1,2}$ Figure 2 shows results of measured vGRF parameters for three different groups during the lifting posture (Group 1: (a) all of the skilled experts; Group 2: (b) all of the unskilled novices; Group 3: (c) all of the experts with MSD.) Three red colored circles and lines indicate mean \pm SD. The horizontal and vertical axes represent the ratio of motion cycle and body mass, respectively. It was shown that there was no big individual difference between motion cycle and the ratio of body mass for Group 1 , but there was a big difference for Group 2 and 3. As you can see results in Figure 2, because the measurement of $\mathrm{CoP}$ had the high feasibility at the logistic workplace, it was possible to self-evaluate characteristics of current own lifting posture through measured data. The recognizing method was used for analyzing the difference of lifting posture between skilled logistic experts and unskilled workers. Thus the proposed method had a high possibility to help in the early detection of poor lifting in order to prevent unskilled workers from MSDs.

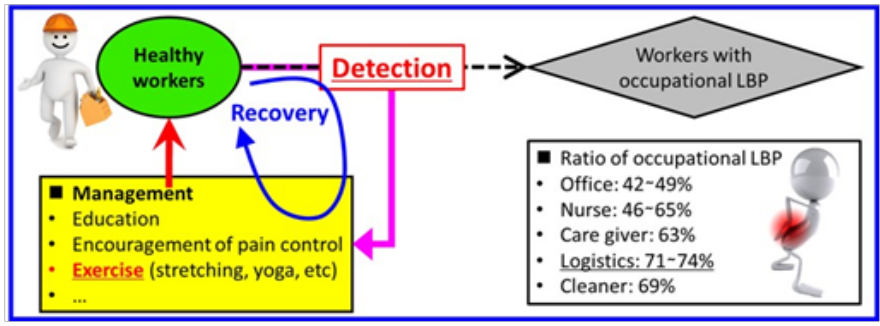

Figure I An importance to recognize the difference in posture between skilled experts and unskilled novices during lifting.

In addition, the use of $\mathrm{CoP}$ trajectories was helpful for recognizing whether there was a difference in MMH between skilled experts and unskilled novices during lifting or not. ${ }^{3}$ If such a difference existed, we could determine what MMH characteristics of experts helped them handle the burden more safely. Our findings showed that most of the skilled experts moved the CoP positions to the opposite side during left asymmetric lifting through modification of the hip position. We inferred that this characteristic of the experts may be involved in lightening the burden on the waist during asymmetric lifting. Recently, our authors have proposed a unique exercise for the logistic workplace which warms up the specific muscles that are activated during lifting by using a musculoskeletal simulation. ${ }^{4}$ Figure 3 shows the results of proposed static posture through all of the activated muscles. Based on the advice of physical therapists, it was possible to formulate these with exercises in which the workers who were the subjects could move without straining themselves. Our exercise enables logistic workers to improve the stretching potential of specific 
muscles, although the limitation of the exercise was not to improve the power potential of muscles. Through clinical tests, it was proven that the stretching ability of muscles might be helpful for improving the balancing ability during lifting. All achievement of research is applied to the actual logistic workplace in order to reduce the ratio of occupational MSD.

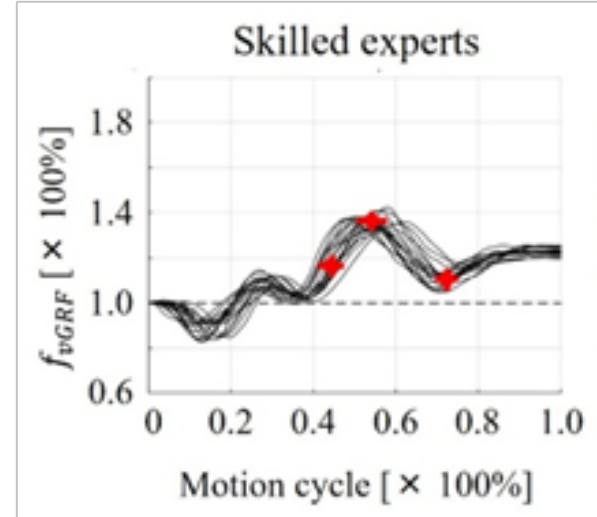

(a) Results of measured vGRFs for all of skilled experts $(n=30)$.

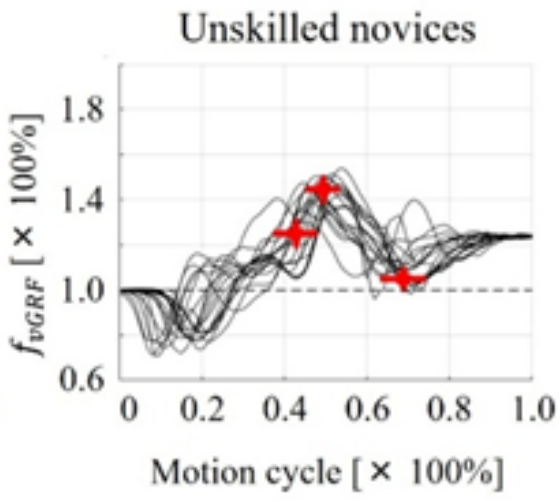

(b) Results of measured vGRFs for all of unskilled novices $(n=30)$.

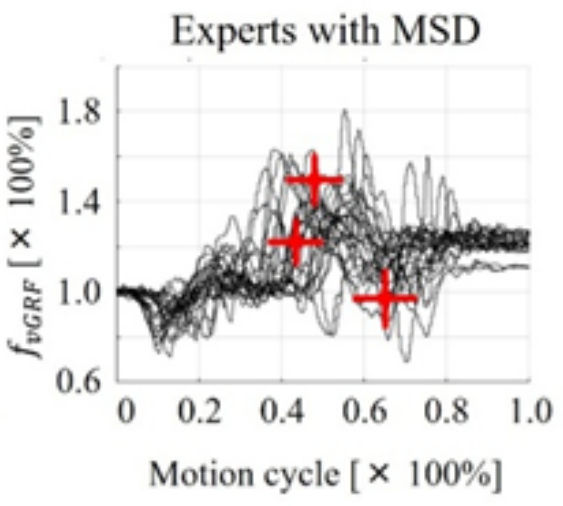

(c) Results of measured vGRFs for all of experts with MSD $(n=42)$.

Figure 2 Results of measured vGRF parameters for three different groups during the lifting posture less than $18 \mathrm{~kg}$. Red colored circles and lines indicated mean $\pm S D$ of $v G R F$ parameters.

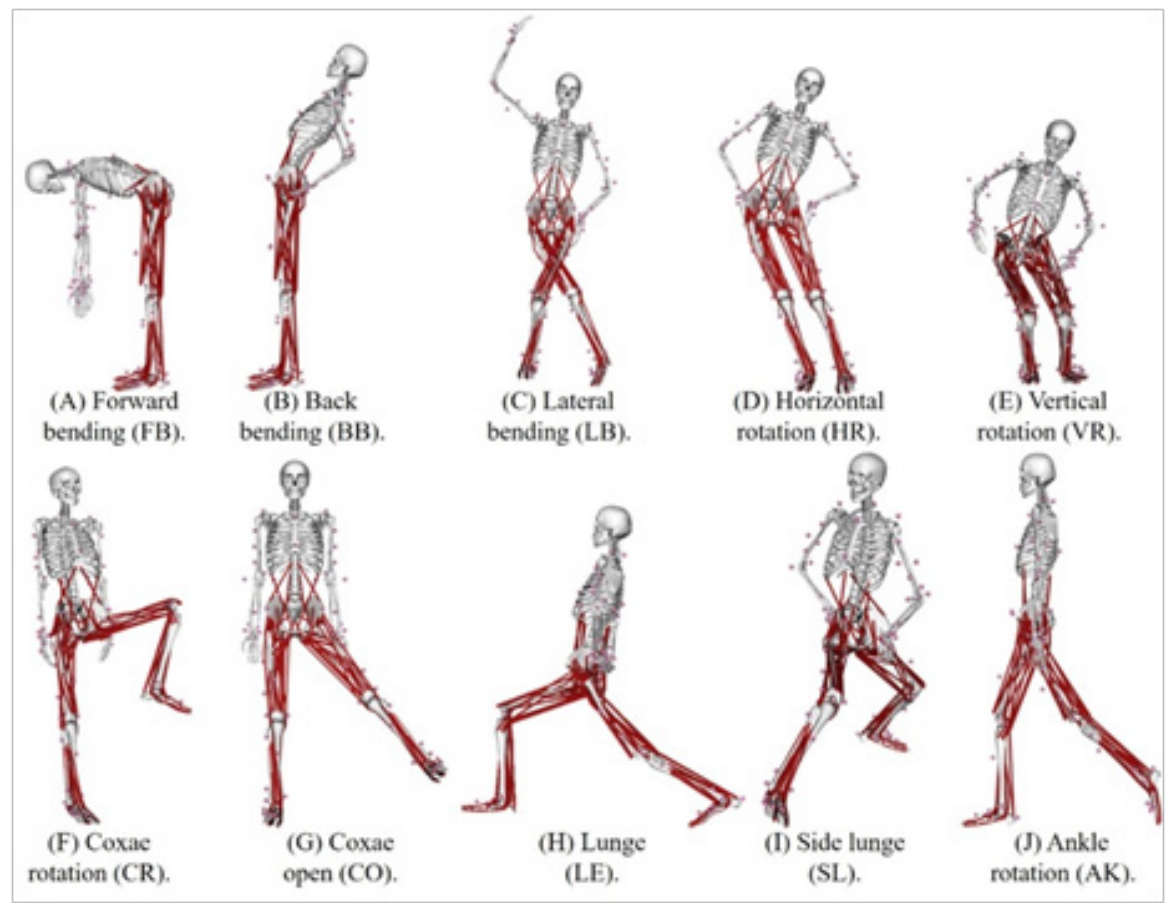

Figure 3 Results of the proposed static posture for all the activated muscles.

\section{Interdisciplinary approach}

Our department is the laboratory for interdisciplinary field of Health Science and Engineering. ${ }^{5}$ Health Science is an interdisciplinary field that helps provide a medical foundation to the public and facilitate disease prevention and recovery. By doing so people can enjoy healthy and quality living. In the future, life-style related diseases such as hypertension and diabetes are expected to rise significantly. Thus the main focus is shifting toward maintaining and enhancing good health as well as preventing diseases. Goals of health promotion science are to investigate methods, implementation and evaluation of health promotion toward individuals, families, groups and different 
environments. The disciplinary convergence help both Health Science and Engineering improve the each academic field and find a new solution for the current problem.

\section{Acknowledgments}

The authors wish to thank the industrial partners involved with the KONOIKE Institute of Technology, KONOIKE Transport Co., Ltd. (Room K708, 7th Floor, 3-1, Ofuka-cho, Kita-ku,Osaka 530-0011, Japan), who helped us find this avenue of research to begin with. They were also of great assistance in developing this particular experiment and preparing this manuscript.

\section{Conflict of interest}

Author declares that there is none of the conflicts.

\section{References}

1. Jeong H, Ohno Y. Symmetric lifting posture recognition of skilled experts with linear discriminant analysis by center-of-pressure velocity. Intel Serv Robotics. 2017. p. 1-10.
2. Jeong H, Kido M, Ohno Y. Linear Discriminant analysis for symmetric lifting recognition of skilled logistic experts by center of pressure trajectory. Conf Proc IEEE Eng Med Biol Soc. 2016. p. 4573-4576.

3. Jeong H, Yamada K, Kido M, et al. Analysis of Difference in Centerof-Pressure Positions Between Experts and Novices During Asymmetric Lifting. IEEE J Transl Eng Health Med. 2016;4:2100311.

4. Watanabe S, Jeong H, Wang T, et al. Proposal of method to prepare musculoskeletal disorders preventive exercise based on musculoskeletal simulation. The International Conference on Biomedical Engineering Innovation. 2017.

5. Yamada K, Ohno Y. Introduction of Laboratory with Interdisciplinary Convergence of Health Science and Engineering in Osaka University. Transactions of the Institute of Systems, Control and Information Engineers. 2016;60(10):451-452.

6. KONOIKE Group website. 2017. 